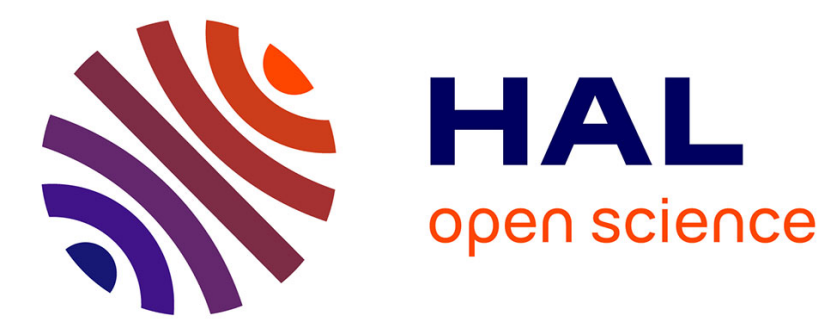

\title{
On transition matrices of Markov chains corresponding to Hamiltonian cycles
}

\author{
Konstantin Avrachenkov, Ali Eshragh, Jerzy A. Filar
}

\section{To cite this version:}

Konstantin Avrachenkov, Ali Eshragh, Jerzy A. Filar. On transition matrices of Markov chains corresponding to Hamiltonian cycles. Annals of Operations Research, 2016, 243 (1-2), pp.19 - 35. 10.1007/s10479-014-1642-2 . hal-01402827

\section{HAL Id: hal-01402827 \\ https://hal.inria.fr/hal-01402827}

Submitted on 25 Nov 2016

HAL is a multi-disciplinary open access archive for the deposit and dissemination of scientific research documents, whether they are published or not. The documents may come from teaching and research institutions in France or abroad, or from public or private research centers.
L'archive ouverte pluridisciplinaire $\mathbf{H A L}$, est destinée au dépôt et à la diffusion de documents scientifiques de niveau recherche, publiés ou non, émanant des établissements d'enseignement et de recherche français ou étrangers, des laboratoires publics ou privés. 


\title{
On transition matrices of Markov chains corresponding to Hamiltonian cycles
}

\author{
Konstantin Avrachenkov* $\quad$ Ali Eshragh ${ }^{\dagger} \quad$ Jerzy A. Filar ${ }^{\ddagger}$
}

November 25, 2016

\begin{abstract}
In this paper, we present some algebraic properties of a particular class of probability transition matrices, namely, Hamiltonian transition matrices. Each matrix $P$ in this class corresponds to a Hamiltonian cycle in a given graph $G$ on $n$ nodes and to an irreducible, periodic, Markov chain. We show that a number of important matrices traditionally associated with Markov chains, namely, the stationary, fundamental, deviation and the hitting time matrix all have elegant expansions in the first $n-1$ powers of $P$, whose coefficients can be explicitly derived. We also consider the resolvent-like matrices associated with any given Hamiltonian cycle and its reverse cycle and prove an identity about the product of these matrices.
\end{abstract}

\section{Introduction}

One of the central concepts in graph theory is the Hamiltonian cycle. Given a graph $G$, a simple path that starts from one node, visits all nodes exactly once and returns to the initial node is called a Hamiltonian cycle or a tour. It should be noted that in this context, terms "Hamiltonian cycle" and "tour" are used, interchangeably. Accordingly, we can define the Hamiltonian Cycle Problem (HCP), which is a well-known problem in graph theory. Particularly, given a directed graph $G$, we are asked to determine whether it contains at least one tour or not. If $G$ contains at least one tour, then the graph is called Hamiltonian and otherwise, that is, if there exists no tour in $G$, it is called a nonHamiltonian graph. In spite of its simple appearance, HCP is an NP-complete problem [7, Chapter 3].

One of the approaches to tackle this problem was proposed in Filar and Krass [5], where the deterministic Hamiltonian cycle problem is converted to a particular average-reward

* INRIA, Sophia Antipolis, France; e-mail: k.avrachenkov@sophia.inria.fr

$\dagger$ School of Mathematical Sciences, The University of Adelaide, Adelaide, SA 5005, Australia; e-mail: ali.eshraghjahromi@adelaide.edu.au

$\ddagger$ School of Computer Science, Engineering and Mathematics, Flinders University, Bedford Park, SA 5042, Australia; e-mail: jerzy.filar@flinders.edu.au 
Markov decision process. In that setting a stationary policy selects a Markov chain whose transitions represent probabilities of travelling on various arcs of a given graph. Of course, if the stationary policy is deterministic, the resulting probability transition matrix has only $0-1$ entries and thus defines a spanning subgraph of the original graph. If, in addition, the latter chain is irreducible the spanning subgraph becomes a Hamiltonian cycle. That was the motivation of a new line of research for the HCP that was summarized in [6]. Essential in this approach are the expansions of key matrices such as the stationary and fundamental matrices associated with various Markov chains induced by the graph $G$ under consideration. Of course, in general, these expansions are infinite series. However, if we restrict ourselves to probability transition matrices induced by Hamiltonian cycles, the preceding expansions reduce to elegant finite series with easily computable coefficients.

The bulk of this paper is devoted to collecting and deriving the above series expansions with the help of Markov chains techniques. To the best of our knowledge the explicit forms of all these series - with closed form expressions for their coefficients - have not been reported elsewhere. In addition, we derive (by similar techniques) an apparently novel identity for the product of the resolvent-like matrices associated with a Hamiltonian cycle and its reverse. Recall that, in an undirected graph, every Hamiltonian cycle is accompanied by a reverse cycle whose probability transition matrix is simply the transpose of that corresponding to the original cycle.

We note that general properties of probability transition matrices and their companions such as fundamental and first hitting time matrices were studied extensively in the classical literature on Markov chains (e.g., see Kemeny and Snell [9]). In more recent years, perturbation properties of these matrices received special attention (e.g., see the book [1] and Hunter [8]).

\section{Hamiltonian Transition Matrices}

Consider a given labeled graph $G$ on $n$ nodes. Suppose $\mathcal{V}=\{1,2, \ldots, n\}$ and $\mathcal{A}$ are, respectively, sets of all nodes and arcs in graph $G$. For each node $i$, we can define two subsets $\mathcal{A}(i)=\{a \in \mathcal{V} \mid(i, a) \in \mathcal{A}\}$ and $\mathcal{B}(i)=\{b \in \mathcal{V} \mid(b, i) \in \mathcal{A}\}$. If $G$ is Hamiltonian, then corresponding to each tour $\tau$ in $G$, we can construct a probability transition matrix, namely a Hamiltonian transition matrix.

Definition 2.1. Suppose the Hamiltonian graph $G$ is given and $\tau$ is a tour in $G$. We say the probability transition matrix $P$ is a "Hamiltonian transition matrix" induced by $\tau$ if it is a 0-1 matrix such that its positive elements correspond to the arcs of the tour $\tau$ in $G$.

For instance, the components of the Hamiltonian transition matrix ${ }^{1} P$ associated with

\footnotetext{
${ }^{1}$ Henceforth, for simplicity, we call Hamiltonian transition matrices just Hamiltonian matrices.
} 
the standard tour " $1 \rightarrow 2 \rightarrow \cdots \rightarrow n \rightarrow 1$ " is

$$
p_{i j}=\left\{\begin{array}{ll}
1 & \text { for } i=1,2, \ldots, n-1, j=i+1 \\
1 & \text { for } i=n, j=1 \\
0 & \text { otherwise }
\end{array} .\right.
$$

Of course, Hamiltonian matrices are special permutation matrices but, in this note, it is their nature as special probability transition matrices of Markov chains that we focus on. Undoubtedly, results we present here could also be derived by other methods that do not exploit Markov chain interpretations but matrices such as the fundamental, deviation and stationary distribution matrix are, perhaps, of most interest to researchers using Markov chains as modelling tools.

It should be noted that in all subsequent sections, we suppose that $P$ is a Hamiltonian matrix induced by a tour in the Hamiltonian graph $G$ on $n$ nodes, unless, otherwise stated. Also, we adopt the convention that matrix $A^{T}$ denotes the transpose of the matrix $A$.

We begin by recalling a number of obvious properties of Hamiltonian matrices that follow directly definitions and Chapman-Kolmogorov equations. These are summarised in the following lemma.

Lemma 2.2. Suppose that the Hamiltonian matrix $P$ is induced by the tour $\tau$ : " $\ell_{0}(=i) \rightarrow \ell_{1} \rightarrow \cdots \rightarrow \ell_{n-1} \rightarrow \ell_{0}$ ".

(i) For values of $r=0,1, \ldots, n-1$, the $i j^{\text {th }}$ component of matrix $P^{r}$ will be equal to

$$
p_{i j}^{(r)}=\delta_{\ell_{r} j},
$$

where $\delta$ is the Kronecker delta.

(ii) The Hamiltonian matrix $P$ has period $n$ and, more generally

$$
P^{k n+r}=P^{r} \quad \text { for } k=0,1,2, \ldots \text { and } r=0,1,2, \ldots, n-1 .
$$

(iii) The eigenvalues of $P$ are the $n$ roots of unity.

\section{Markov Chains}

According to Definition 2.1, any Hamiltonian matrix is a probability transition matrix. This implies that we can derive all properties of probability transition matrices for them, as well.

Remark 3.1. According to Lemma 2.2, the Markov chain defined by each Hamiltonian matrix is irreducible. This indicates that for each Hamiltonian matrix, there exists a unique stationary distribution, as well as, Cesaro limit matrix. Moreover, it is easy to see that the former follows the uniform distribution. 


\section{Proposition 3.2. For Hamiltonian matrix P,}

(i) the Cesaro limit matrix, denoted by $P^{*}$, is equal to $\frac{1}{n} J$, where $J$ is a square matrix with all entries equal to 1 ;

(ii) matrix $P^{*}$ can be expressed in a finite sum of powers of $P$ as follows

$$
P^{*}=\frac{1}{n} \sum_{r=0}^{n-1} P^{r} .
$$

\section{Proof.}

(i) This result can be obtained immediately from Remark 3.1.

(ii) By considering part (i), we just need to show that $\sum_{r=0}^{n-1} P^{r}=J$. Let us suppose that the Hamiltonian matrix $P$ is associated with the tour " $\ell_{0}(=i) \rightarrow \ell_{1} \rightarrow \cdots \rightarrow$ $\ell_{n-1} \rightarrow \ell_{0}$ ". Now, by considering Lemma 2.2, the $i j^{\text {th }}$ component of summation $\sum_{r=0}^{n-1} P^{r}$ is equal to

$$
\sum_{r=0}^{n-1} p_{i j}^{(r)}=\sum_{r=0}^{n-1} \delta_{\ell_{r} j}=\sum_{k=1}^{n} \delta_{k j}=1
$$

One may be interested in defining the (first) hitting time matrix $H$, such that its $i j^{\text {th }}$ component, $h_{i j}$, identifies the hitting time of node $j$, starting from node $i$ and following Markov chain defined by the Hamiltonian matrix $P$. From Lemma 2.2, it is readily seen that

$$
h_{i j}=\min _{0 \leq r \leq n-1}\left\{r \mid p_{i j}^{(r)}>0\right\} .
$$

Accordingly, we can express the hitting time matrix in terms of the first $n-1$ powers of $P$.

Lemma 3.3. For a Hamiltonian matrix $P$, the hitting time matrix can be expressed in terms of the first $n-1$ powers of $P$ as follows

$$
H=\sum_{r=0}^{n-1} r P^{r} .
$$

Proof. Without loss of generality, suppose that the Hamiltonian matrix $P$ is associated with tour " $\ell_{0}(=i) \rightarrow \ell_{1} \rightarrow \cdots \rightarrow \ell_{k}(=j) \rightarrow \cdots \rightarrow \ell_{n-1} \rightarrow \ell_{0}$ ", that is, $h_{i j}=k$, for $i, j \in \mathcal{V}$. By considering Lemma 2.2, the $i j^{t h}$ element of the right hand side of (2) is equal to

$$
\sum_{r=0}^{n-1} r \delta_{\ell_{r} j}=k=h_{i j}
$$


Surprisingly, irrespective of the Hamiltonian cycle that defines matrix $P$, we can show that the inverse of corresponding hitting time matrix $H$ can be expressed in sum of finite powers of $P$, as well. For this purpose, firstly, we need to show the following lemma.

Lemma 3.4. Consider a Hamiltonian matrix $P$ and its corresponding hitting time matrix H. We have

(i) $H P=H-J+n I$;

(ii) $H J=\frac{n(n-1)}{2} J$.

\section{Proof.}

(i) By considering Lemma 3.3, Lemma 2.2 and Proposition 3.2, we will have

$$
\begin{aligned}
H P & =\sum_{r=0}^{n-1} r P^{r+1}=\sum_{r=1}^{n}(r-1) P^{r} \\
& =\sum_{r=1}^{n} r P^{r}-\sum_{r=1}^{n} P^{r}=\left(\sum_{r=0}^{n-1} r P^{r}+n I\right)-\sum_{r=0}^{n-1} P^{r} \\
& =H-J+n I .
\end{aligned}
$$

(ii) It is clear that $J=\mathbf{1 1}^{T}$. The $i^{\text {th }}$ component of vector $H \mathbf{1}$ is equal to

$$
\sum_{k=1}^{n} h_{i k}=\sum_{r=0}^{n-1} r=\frac{n(n-1)}{2} .
$$

This implies that $H \mathbf{1}=\frac{n(n-1)}{2} \mathbf{1}$, and accordingly, $H \mathbf{1 1} \mathbf{1}^{T}=\frac{n(n-1)}{2} \mathrm{~J}$.

Proposition 3.5. Consider a Hamiltonian matrix $P$ and its corresponding hitting time matrix $H$. The inverse of matrix $H$ exists and can be expressed as a finite sum of powers of matrix $P$ as follows:

$$
H^{-1}=-\frac{n^{2}-n-2}{n^{2}(n-1)} I+\frac{n^{2}-n+2}{n^{2}(n-1)} P+\frac{2}{n^{2}(n-1)} \sum_{r=2}^{n-1} P^{r} .
$$

Proof. Obvjously, if we can show that the product of matrix $H$ and the right hand side of (3) is equal to $I$, then, simultaneously, we have shown that the inverse of matrix $H$ exists and also equal to (3). For this purpose, we denote the right hand side of (3) by $\Gamma$ and rewrite it as follows:

$$
\Gamma=\frac{2}{n^{2}(n-1)} \sum_{r=0}^{n-1} P^{r}-\frac{1}{n} I+\frac{1}{n} P,
$$


which is, from Proposition 3.2, equivalent to

$$
\Gamma=\frac{2}{n^{2}(n-1)} \mathbf{1 1}^{T}-\frac{1}{n} I+\frac{1}{n} P .
$$

Now, by exploiting Lemma 3.4, we will have

$$
\begin{aligned}
H \Gamma & =\frac{2}{n^{2}(n-1)} H J-\frac{1}{n} H+\frac{1}{n} H P \\
& =\frac{2}{n^{2}(n-1)}\left(\frac{n(n-1)}{2} J\right)-\frac{1}{n} H+\frac{1}{n}(H-J+n I) \\
& =I .
\end{aligned}
$$

Consequently, the $H^{-1}$ exists and is equal to $\Gamma$.

\section{Matrices Associated with Markov Chains}

In this section, we, mainly, want to derive explicit formulation for classical matrices in Markov decision processes induced by Hamiltonian matrices. At the outset, we recall such matrices from [10, Appendix A]. It must be noted that following definitions hold not only for Hamiltonian matrices, but also for any probability transition matrix $P$.

The fundamental matrix is denoted by $F$ and defined as follows

$$
F:=\left(I-P+P^{*}\right)^{-1},
$$

where $P$ is a probability transition matrix and $P^{*}$ is its Cesaro limit matrix. Accordingly, we can define the deviation matrix through

$$
D:=F-P^{*} .
$$

The main properties of these matrices asserted in the following theorem (e.g., see $[10$, Appendix A]):

Theorem 4.1. If $P$ is a probability transition matrix with Cesaro limit matrix $P^{*}$, then (i) $P^{* 2}=P^{*}$; (ii) $P P^{*}=P^{*} P=P^{*}$; (iii) $\left(P-P^{*}\right)^{k}=P^{k}-P^{*}$ for $k=1,2, \ldots$; (iv) $F P^{*}=P^{*} F=P^{*}$; (v) $D P^{*}=P^{*} D=0$.

Based on mentioned definitions and notations, we want to know if we can express the fundamental matrix, as well as, the deviation matrix induced by a Hamiltonian matrix $P$ as a finite sum of powers of $P$.

Proposition 4.2. For a Hamiltonian matrix $P$, the corresponding fundamental matrix $F$ and deviation matrix $D$, can be expressed as follows:

$$
\begin{aligned}
& F=\sum_{r=0}^{n-1} \frac{n+1-2 r}{2 n} P^{r} \\
& D=\sum_{r=0}^{n-1} \frac{n-1-2 r}{2 n} P^{r} .
\end{aligned}
$$


Proof. It is apparent from (4) that, if we can show $\left(I-P+P^{*}\right) \sum_{r=0}^{n-1} \frac{n+1-2 r}{2 n} P^{r}=I$, then we shall conclude that (6) holds. Hence, by exploiting part (ii) of Theorem 4.1 and Proposition 3.2, we will have

$$
\begin{aligned}
(I-P & \left.+P^{*}\right) \sum_{r=0}^{n-1} \frac{n+1-2 r}{2 n} P^{r} \\
& =\sum_{r=0}^{n-1} \frac{n+1-2 r}{2 n} P^{r}-\sum_{r=0}^{n-1} \frac{n+1-2 r}{2 n} P^{r+1}+\sum_{r=0}^{n-1} \frac{n+1-2 r}{2 n} P^{*} \\
& =\sum_{r=0}^{n-1} \frac{n+1-2 r}{2 n} P^{r}-\sum_{r=1}^{n} \frac{n+3-2 r}{2 n} P^{r}+P^{*} \\
& =\frac{n-1}{n} I-\frac{1}{n} \sum_{r=1}^{n-1} P^{r}+P^{*} \\
& =\frac{n-1}{n} I-\left(P^{*}-\frac{1}{n} I\right)+P^{*} \\
& =I .
\end{aligned}
$$

Now, we just need to utilize (5), as well as Proposition 3.2 to show (7) holds.

$$
\begin{aligned}
D & =F-P^{*}=\sum_{r=0}^{n-1} \frac{n+1-2 r}{2 n} P^{r}-\frac{1}{n} \sum_{r=0}^{n-1} P^{r} \\
& =\sum_{r=0}^{n-1} \frac{n-1-2 r}{2 n} P^{r} .
\end{aligned}
$$

Corollary 4.3. If $P$ is a Hamiltonian matrix, then the $i j^{\text {th }}$ component of its corresponding fundamental matrix $F$ is equal to

$$
\frac{n+1-2 h_{i j}}{2 n}
$$

Proof. Recalling Lemma 3.3, we know that for a fixed pair $(i, j)$, all components $p_{i j}^{(r)}$ are equal to zero, except for $r=h_{i j}$. More precisely, $p_{i j}^{(r)}=\delta_{r h_{i j}}$. Therefore, from Proposition 4.2 we can see that the $i j^{\text {th }}$ component of the fundamental matrix $F$ is equal to $\frac{n+1-2 h_{i j}}{2 n}$.

Remark 4.4. Borkar et. al. [2] exploited the optional sampling theorem to develop explicit formulae for entries of a fundamental matrix $F$ induced by a doubly stochastic matrix $x^{2}$. More precisely, they expressed each entry of $F$ in terms of the hitting times. As each Hamiltonian matrix is also a doubly stochastic matrix, naturally, (8) coincides with results in [2].

\footnotetext{
${ }^{2}$ A matrix is called doubly stochastic, if its entries are non-negative, as well as, all rows and columns sum up 1.
} 
Corollary 4.5. For a given Hamiltonian matrix, the powers of the corresponding fundamental matrix as well as deviation matrix can be expressed as a sum of the first $n-1$ powers of matrix $P$, that is,

$$
\begin{aligned}
F^{k} & =\sum_{r=0}^{n-1} f_{r}^{k} P^{r} \quad \text { for } k=1,2, \ldots \\
D^{k} & =\sum_{r=0}^{n-1} d_{r}^{k} P^{r} \quad \text { for } k=1,2, \ldots,
\end{aligned}
$$

where $f_{r}^{k}$ and $d_{r}^{k}$ are real coefficients.

Proof. As Proposition 4.2 expresses both $F$ and $D$ as a sum of the first $n$ powers of matrix $P$, and also, due the periodicity of matrix $P$ mentioned in Lemma 2.2, the existence of finite series in (9) and (10) will yield.

Now, one may be interested in finding coefficients $f_{r}^{k}$ and $d_{r}^{k}$. Obviously, from Proposition 4.2, we know that,

$$
f_{r}^{1}=\frac{n+1-2 r}{2 n} ; \quad d_{r}^{1}=\frac{n-1-2 r}{2 n},
$$

for $r=0,1, \ldots, n-1$. Hence, if we can find a recursive formulation for them, we will be able to calculate them, directly.

Proposition 4.6. For a given Hamiltonian matrix $P$ and its corresponding fundamental matrix, the following hold for $k=1,2, \ldots$ :

(i) $\sum_{r=0}^{n-1} f_{r}^{k}=1$

(ii) $\left\{\begin{array}{l}f_{0}^{k+1}=f_{0}^{k}+\frac{1}{n} \sum_{s=1}^{n-1} s f_{s}^{k}-\frac{n-1}{2 n} \\ f_{r}^{k+1}=f_{0}^{k+1}+\sum_{s=1}^{r} f_{s}^{k}-\frac{r}{n} \text { for } r=1,2, \ldots, n-1\end{array}\right.$.

Proof.

(i) If we multiply both sides of Equation (9) by $P^{*}$ and utilize parts (ii) and (iv) of Theorem 4.1, we will have

$$
P^{*}=\sum_{r=0}^{n-1} f_{r}^{k} P^{*} \text { for } k=1,2, \ldots,
$$

or equivalently,

$$
\sum_{r=0}^{n-1} f_{r}^{k}=1 \text { for } k=1,2, \ldots
$$


(ii) We start with the trivial equation $F^{k}=F^{k+1} F^{-1}$ for $k=1,2, \ldots$. By definition of $F$, we can write it as $F^{k}=F^{k+1}\left(I-P+P^{*}\right)$. Now, analogous to the previous part, we can simplify the latter as follows:

$$
\sum_{r=0}^{n-1} f_{r}^{k} P^{r}=\sum_{r=0}^{n-1} f_{r}^{k+1} P^{r}-\sum_{r=0}^{n-1} f_{r}^{k+1} P^{r+1}+\sum_{r=0}^{n-1} f_{r}^{k+1} P^{*} .
$$

Now by considering part (i) of this proposition, as well as, part (ii) of Proposition 3.2 , we will have

$$
\sum_{r=0}^{n-1} f_{r}^{k} P^{r}=\sum_{r=0}^{n-1} f_{r}^{k+1} P^{r}-\sum_{r=0}^{n-1} f_{r}^{k+1} P^{r+1}+\frac{1}{n} \sum_{r=0}^{n-1} P^{r}
$$

or, equivalently,

$$
f_{0}^{k} I+\sum_{r=1}^{n-1} f_{r}^{k} P^{r}=\left(f_{0}^{k+1}-f_{n-1}^{k+1}-\frac{1}{n}\right) I+\sum_{r=1}^{n-1}\left(f_{r}^{k+1}-f_{r-1}^{k+1}+\frac{1}{n}\right) P^{r} .
$$

Similar to the previous part, by equating coefficients of the same powers of $P$ in both sides of (11), we can derive the following recursive equation:

$$
f_{r}^{k+1}=f_{0}^{k+1}+\sum_{s=1}^{r} f_{s}^{k}-\frac{r}{n} \text { for } r=1,2, \ldots, n-1 .
$$

Now, by substituting (12) into equation $\sum_{r=0}^{n-1} f_{r}^{k+1}=1$, grouping terms involving $f_{0}^{k+1}$ on the left side, we will obtain

$$
\begin{aligned}
f_{0}^{k+1} & =\frac{n+1}{2 n}-\frac{1}{n} \sum_{r=1}^{n-1} \sum_{s=1}^{r} f_{s}^{k}=\frac{n+1}{2 n}-\frac{1}{n} \sum_{s=1}^{n-1} \sum_{r=s}^{n-1} f_{s}^{k} \\
& =\frac{n+1}{2 n}-\frac{1}{n} \sum_{s=1}^{n-1}(n-s) f_{s}^{k}=\frac{n+1}{2 n}-\frac{1}{n}\left(n \sum_{s=1}^{n-1} f_{s}^{k}-\sum_{s=1}^{n-1} s f_{s}^{k}\right) \\
& =\frac{n+1}{2 n}-\left(1-f_{0}^{k}\right)+\frac{1}{n} \sum_{s=1}^{n-1} s f_{s}^{k}=f_{0}^{k}+\frac{1}{n} \sum_{s=1}^{n-1} s f_{s}^{k}-\frac{n-1}{2 n} .
\end{aligned}
$$

In order to derive analogous recursive formula for coefficients $d_{r}^{k}$, we first find a linear relationship between coefficients $d_{r}^{k}$ and $f_{r}^{k}$, and then, exploit Proposition 4.6.

Lemma 4.7. For a given Hamiltonian matrix $P$ and the corresponding fundamental and deviation matrices,

$$
d_{r}^{k}=f_{r}^{k}-\frac{1}{n} \text { for } r=0,1, \ldots, n-1, k=1,2, \ldots
$$


Proof. Recalling definition of deviation matrix $D$ given in (5), we have $D=F-P^{*}$. This implies that

$$
D^{k}=\left(F-P^{*}\right)^{k}=\sum_{s=0}^{k}\left(\begin{array}{l}
n \\
s
\end{array}\right) F^{s}\left(-P^{*}\right)^{k-s}=F^{k}-\sum_{s=1}^{k}\left(\begin{array}{c}
n \\
s
\end{array}\right)(-1)^{k-s} P^{*}=F^{k}-P^{*} .
$$

Therefore, by considering (9), (10) and Proposition 3.2, we have

$$
\sum_{r=0}^{n-1} d_{r}^{k} P^{r}=\sum_{r=0}^{n-1} f_{r}^{k} P^{r}-\frac{1}{n} \sum_{r=0}^{n-1} P^{r}=\sum_{r=0}^{n-1}\left(f_{r}^{k}-\frac{1}{n}\right) P^{r}
$$

Now, by equating coefficient of the same powers of $P$ in both sides of (13), we derive

$$
d_{r}^{k}=f_{r}^{k}-\frac{1}{n} .
$$

Corollary 4.8. For a given Hamiltonian matrix $P$ and the corresponding deviation matrix, the following hold for $k=1,2, \ldots$ :

(i) $\sum_{r=0}^{n-1} d_{r}^{k}=0$,

(ii) $\left\{\begin{array}{l}d_{0}^{k+1}=d_{0}^{k}+\frac{1}{n} \sum_{s=0}^{n-1} s d_{s}^{k} \\ d_{r}^{k+1}=d_{0}^{k+1}+\sum_{s=1}^{r} d_{s}^{k} \text { for } r=1,2, \ldots, n-1\end{array}\right.$.

Proof. Both two parts (i) and (ii) can be easily found by replacing $f_{r}^{k}$ with $d_{r}^{k}+\frac{1}{n}$ in parts (i)-(ii) of Proposition 4.6.

Another matrix that plays an essential role in the theory of discounted Markov decision processes we shall name the $\beta$-resolvent matrix. More precisely, if $P$ is a probability transition matrix and $\beta \in(0,1)$ is a discount factor, the $\beta$-resolvent matrix, $R(\beta)$, is defined as follows:

$$
R(\beta):=(I-\beta P)^{-1}
$$

Analogous to the fundamental and deviation matrices, for the special case of Hamiltonian matrix $P$, we can derive a finite sum of powers of matrix $P$ for $R(\beta)$, as expressed in the following proposition.

Proposition 4.9. If $P$ is a Hamiltonian matrix, then for any discount factor $\beta \in(0,1)$,

$$
R(\beta)=\frac{1}{1-\beta^{n}} \sum_{r=0}^{n-1} \beta^{r} P^{r}
$$


Proof. By exploiting Lemma 2.2, we will have

$$
R(\beta)=\sum_{i=0}^{\infty} \beta^{i} P^{i}=\sum_{s=0}^{\infty} \sum_{r=0}^{n-1} \beta^{s n+r} P^{s n+r}=\sum_{r=0}^{n-1} \sum_{s=0}^{\infty} \beta^{s n+r} P^{r}=\frac{1}{1-\beta^{n}} \sum_{r=0}^{n-1} \beta^{r} P^{r} .
$$

Corollary 4.10. If $P$ is a Hamiltonian matrix, the $i j^{\text {th }}$ component of its $\beta$-resolvent matrix $R(\beta)$ is equal to

$$
\frac{\beta^{h_{i j}}}{1-\beta^{n}}
$$

Proof. Recalling Lemma 3.3, we know that for a fix pair $(i, j)$, all components $p_{i j}^{(r)}$ are equal to zero, except for $r=h_{i j}$. More precisely, $p_{i j}^{(r)}=\delta_{r} h_{i j}$. Therefore, from Proposition 4.9 , we can see that the $i j^{\text {th }}$ component of the $\beta$-resolvent matrix is equal to $\frac{\beta^{h} i j}{1-\beta^{n}}$.

\section{$5 \quad$ Undirected Graphs}

Let us consider the class of undirected graphs. Clearly, if a given undirected graph $G$ is Hamiltonian, then corresponding to each tour $\tau:$ " $\ell_{0} \rightarrow \ell_{1} \rightarrow \cdots \rightarrow \ell_{n-1} \rightarrow \ell_{0}$ " in $G$, its reverse, $\tau^{\mathcal{R}}:$ " $\ell_{0} \rightarrow \ell_{n-1} \rightarrow \cdots \rightarrow \ell_{1} \rightarrow \ell_{0}$ ", also exists in $G$. Accordingly, if the Hamiltonian matrix $P$ is corresponding to tour $\tau$, then its transpose, $P^{T}$ is corresponding to the reverse tour $\tau^{\mathcal{R}}$. Hence, the following result can be obtained, immediately:

$$
\left(P^{T}\right)^{r}=P^{n-r} \text { for } r=0,1, \ldots, n-1 .
$$

It should be noted that in this section, our results concern undirected graphs, unless otherwise is mentioned.

We can also derive the following result for hitting time matrix in an undirected graph.

Lemma 5.1. Consider the Hamiltonian matrix $P$ corresponding to tour $\tau$ and its corresponding hitting time matrix $H$. If $H^{\mathcal{R}}$ denotes the hitting time matrix associated with the reverse tour $\tau^{\mathcal{R}}$, we will have

(i) $H^{\mathcal{R}}=\sum_{r=1}^{n-1}(n-r) P^{r}$;

(ii) $H+H^{\mathcal{R}}=n(J-I)$.

\section{Proof.}


(i) By utilizing Lemma 3.3, as well as, Equation (15), we will have

$$
\begin{aligned}
H^{\mathcal{R}} & =\sum_{r=0}^{n-1} r\left(P^{T}\right)^{r}=\sum_{r=1}^{n-1} r\left(P^{T}\right)^{r} \\
& =\sum_{r=1}^{n-1} r P^{n-r}=\sum_{r=1}^{n-1}(n-r) P^{r} \\
& =\sum_{r=1}^{n-1}(n-r) P^{r} .
\end{aligned}
$$

(ii) Now, by considering part (i), we should have

$$
\begin{aligned}
H+H^{\mathcal{R}} & =\sum_{r=0}^{n-1} r P^{r}+\sum_{r=1}^{n-1}(n-r) P^{r} \\
& =\sum_{r=1}^{n-1} n P^{r}=n \sum_{r=1}^{n-1} P^{r} \\
& =n(J-I),
\end{aligned}
$$

where the last equality comes from Proposition 3.2.

Consider the Hamiltonian matrix $P$ and its corresponding $\beta$-resolvent matrix $R(\beta)$. Let $R^{\mathcal{R}}(\beta)$ denote the $\beta$-resolvent matrix associated with the reverse tour corresponding to $P$, that is, $R^{\mathcal{R}}(\beta)=\left(I-\beta P^{T}\right)^{-1}$. The following proposition expresses an interesting relationship between them. However, we need the following lemma to prove it, in advance.

Lemma 5.2. For a Hamiltonian matrix $P$, the $i j^{\text {th }}$ component of its corresponding matrix $R^{\mathcal{R}}(\beta)$ is equal to

$$
r_{i j}^{\mathcal{R}}(\beta)=\frac{\beta^{h_{j i}}}{1-\beta^{n}}
$$

Proof. By considering Corollary 4.10,

$$
r_{i j}^{\mathcal{R}}(\beta)=\frac{\beta^{h_{i j}^{\mathcal{R}}}}{1-\beta^{n}},
$$

where $h_{i j}^{\mathcal{R}}$ is the length of the path from $i$ to $j$ in the reverse tour. Apparently, such length is equal to the length of the path from $j$ to $i$ in the direct tour, i.e., $h_{j i}$.

Proposition 5.3. If $P$ is a Hamiltonian matrix corresponding to a tour $\tau$, then for any discount factor $\beta \in(0,1)$,

$$
R(\beta) R^{\mathcal{R}}(\beta)=\frac{1}{1-\beta^{2}}\left(R(\beta)+R^{\mathcal{R}}(\beta)-I\right) .
$$


Proof. Recalling Corollary 4.10 and Lemma 5.2, it is readily seen that the right hand side of (16) is equal to

$$
\frac{1}{1-\beta^{2}}\left(\frac{\beta^{h_{i j}}}{1-\beta^{n}}+\frac{\beta^{h_{j i}}}{1-\beta^{n}}-\delta_{i j}\right)
$$

Therefore, we just need to show that the corresponding components of the left hand side are equal to (17), as well. The $i^{\text {th }}$ diagonal element of the left hand side is equal to

$$
\begin{aligned}
{\left[R(\beta) R^{\mathcal{R}}(\beta)\right]_{i i} } & =\sum_{k=1}^{n} r_{i k}(\beta) r_{k i}^{\mathcal{R}}(\beta)=\sum_{k=1}^{n}\left(\frac{\beta^{h_{i k}}}{1-\beta^{n}}\right)\left(\frac{\beta^{h_{i k}}}{1-\beta^{n}}\right) \\
& =\frac{1}{\left(1-\beta^{n}\right)^{2}} \sum_{k=1}^{n} \beta^{2 h_{i k}}=\frac{1}{\left(1-\beta^{n}\right)^{2}} \sum_{s=0}^{n-1} \beta^{2 s} \\
& =\frac{1}{\left(1-\beta^{n}\right)^{2}}\left(\frac{1-\beta^{2 n}}{1-\beta^{2}}\right)=\frac{1+\beta^{n}}{\left(1-\beta^{n}\right)\left(1-\beta^{2}\right)} \\
& =\frac{1}{1-\beta^{2}}\left(\frac{1}{1-\beta^{n}}+\frac{1}{1-\beta^{n}}-1\right) \\
& =\frac{1}{1-\beta^{2}}\left(\frac{\beta^{h_{i i}}}{1-\beta^{n}}+\frac{\beta^{h_{i i}}}{1-\beta^{n}}-\delta_{i i}\right) .
\end{aligned}
$$

Analogously, let us consider the $i j^{\text {th }}$ component of $R(\beta) R^{\mathcal{R}}(\beta)$ for $j \neq i$. It follows that

$$
\begin{aligned}
{\left[R(\beta) R^{\mathcal{R}}(\beta)\right]_{i j} } & =\sum_{k=1}^{n} r_{i k}(\beta) r_{k j}^{\mathcal{R}}(\beta)=\sum_{k=1}^{n}\left(\frac{\beta^{h_{i k}}}{1-\beta^{n}}\right)\left(\frac{\beta^{h_{j k}}}{1-\beta^{n}}\right) \\
& =\frac{1}{\left(1-\beta^{n}\right)^{2}} \sum_{k=1}^{n} \beta^{h_{i k}+h_{j k}} .
\end{aligned}
$$

Now, let us partition the set of all nodes $\mathcal{V}$ into two subsets $\mathcal{V}_{1}$ and $\mathcal{V}_{2}$, such that the former comprises all nodes in tour $\tau$, starting from $i$ up to one before $j$ and the latter is its complement. Obviously, the cardinality of subsets $\mathcal{V}_{1}$ and $\mathcal{V}_{2}$ are equal to $h_{i j}$ and $h_{j i}$, 
respectively. Hence, (18) is equal to

$$
\begin{aligned}
{\left[R(\beta) R^{\mathcal{R}}(\beta)\right]_{i j} } & =\frac{1}{\left(1-\beta^{n}\right)^{2}}\left(\sum_{k \in \mathcal{V}_{1}} \beta^{h_{i k}+h_{j k}}+\sum_{k \in \mathcal{V}_{2}} \beta^{h_{i k}+h_{j k}}\right) \\
& =\frac{1}{\left(1-\beta^{n}\right)^{2}}\left(\sum_{k \in \mathcal{V}_{1}} \beta^{2 h_{i k}+h_{j i}}+\sum_{k \in \mathcal{V}_{2}} \beta^{h_{i j}+2 h_{j k}}\right) \\
& =\frac{1}{\left(1-\beta^{n}\right)^{2}}\left(\beta^{h_{j i}} \sum_{k \in \mathcal{V}_{1}} \beta^{2 h_{i k}}+\beta^{h_{i j}} \sum_{k \in \mathcal{V}_{2}} \beta^{2 h_{j k}}\right) \\
& =\frac{1}{\left(1-\beta^{n}\right)^{2}}\left(\beta^{h_{j i}} \sum_{s=0}^{h_{i j}} \beta^{2 s}+\beta^{h_{i j}} \sum_{s=0}^{h_{j i}} \beta^{2 s}\right) \\
& =\frac{1}{\left(1-\beta^{n}\right)^{2}}\left(\beta^{h_{j i}} \frac{1-\beta^{2 h_{i j}}}{1-\beta^{2}}+\beta^{h_{i j}} \frac{1-\beta^{2 h_{j i}}}{1-\beta^{2}}\right) \\
& =\frac{1}{\left(1-\beta^{n}\right)^{2}\left(1-\beta^{2}\right)}\left(\beta^{h_{j i}}-\beta^{h_{j i}+2 h_{i j}}+\beta^{h_{i j}}-\beta^{h_{i j}+2 h_{j i}}\right) .
\end{aligned}
$$

Now, by exploiting the fact that $h_{i j}+h_{j i}=n$, we will have

$$
\begin{aligned}
{\left[R(\beta) R^{\mathcal{R}}(\beta)\right]_{i j} } & =\frac{1}{\left(1-\beta^{n}\right)^{2}\left(1-\beta^{2}\right)}\left(\beta^{h_{j i}}-\beta^{n+h_{i j}}+\beta^{h_{i j}}-\beta^{n+h_{j i}}\right) \\
& =\frac{1}{\left(1-\beta^{n}\right)^{2}\left(1-\beta^{2}\right)}\left(\beta^{h_{i j}}\left(1-\beta^{n}\right)+\beta^{h_{j i}}\left(1-\beta^{n}\right)\right) \\
& =\frac{1}{\left(1-\beta^{2}\right)}\left(\frac{\beta^{h_{i j}}}{1-\beta^{n}}+\frac{\beta^{h_{j i}}}{1-\beta^{n}}\right) \\
& =\frac{1}{\left(1-\beta^{2}\right)}\left(r_{i j}+r_{i j}^{\mathcal{R}}-\delta_{i j}\right) .
\end{aligned}
$$

\section{A New Parameter-Free Polytope}

In this section we introduce an application of theoretical results presented in previous sections to design a heuristic algorithm to determine the non-Hamiltonicity of a given graph. It is known that each Discounted Markov Decision Process (DMDP) is associated with the system of linear equations (e.g., see [6, Chapter 2] and [10, Chapter 6]):

$$
\boldsymbol{x}_{f}(\beta)^{T}\left(I-\beta P_{f}\right):=\gamma^{T},
$$

or, equivalently,

$$
\sum_{i=1}^{n} x_{i}\left(\delta_{i j}-\beta p_{i j}\right)=\gamma_{j} \text { for } j=1,2, \ldots, n
$$


where $\boldsymbol{\gamma}$ is the initial distribution. Furthermore, by assuming $\boldsymbol{x}_{f}(\beta)>\mathbf{0}$ and defining $x_{i a}:=x_{i} f(i, a)$, we can rewrite the left hand side of (20) as below:

$$
\begin{aligned}
\sum_{i=1}^{n} x_{i}\left(\delta_{i j}-\beta p_{i j}\right) & =\sum_{i=1}^{n} x_{i} \sum_{a \in \mathcal{A}(i)}\left(\delta_{i j}-\beta p_{i j}(a)\right) f(i, a)=\sum_{i=1}^{n} x_{i} \sum_{a \in \mathcal{A}(i)}\left(\delta_{i j}-\beta p_{i j}(a)\right) \frac{x_{i a}}{x_{i}} \\
& =\sum_{a \in \mathcal{A}(j)} x_{j a}-\beta \sum_{b \in \mathcal{B}(j)} x_{b j} .
\end{aligned}
$$

Thus, (20) is simplified as below:

$$
\sum_{a \in \mathcal{A}(j)} x_{j a}-\beta \sum_{b \in \mathcal{B}(j)} x_{b j}=\gamma_{j} \text { for } j=1,2, \ldots, n .
$$

In 2000, Feinberg [4] converted HCP to a class of DMDPs and showed that HCP can be reduced to the problem of finding a feasible deterministic policy for a DMDP with constraints by proving the following theorem:

Theorem 6.1. Consider the following linear constraints regarding the given graph $G$ :

$$
\begin{aligned}
\sum_{a \in \mathcal{A}(i)} y_{i a}-\beta \sum_{b \in \mathcal{B}(i)} y_{b i} & =\delta_{i 1} \quad \text { for all } i \in \mathcal{V} \\
\sum_{a \in \mathcal{A}(1)} y_{1 a} & =\frac{1}{1-\beta^{n}} \\
y_{i a} & \geq 0 \text { for all } i \in \mathcal{V}, a \in \mathcal{A}(i)
\end{aligned}
$$

where $\beta$ is a fixed discount factor chosen arbitrarily from interval $[0,1)$. The graph $G$ is Hamiltonian if and only if the linear Constraints (22)-(24) have at least one feasible solution $\boldsymbol{y}$ corresponding to a deterministic policy the so-called "Hamiltonian policy". That is, if for each $i \in \mathcal{V}$, there is exactly one $a \in \mathcal{A}(i)$ such that, $y_{i a}>0$. In other words,

$$
f(i, a):=\frac{y_{i a}}{\sum_{j \in \mathcal{A}(i)} y_{i j}} \in\{0,1\} ; \forall i \in \mathcal{V}, a \in \mathcal{A}(i) .
$$

If such solution is found, then the corresponding deterministic policy $f$ traces out a Hamiltonian cycle in $G$.

As an example, let us construct Constraints (22)-(24) for the graph given in Figure 1, as below:

$$
\begin{aligned}
y_{12}+y_{13}+y_{14}-\beta y_{21}-\beta y_{41} & =1 \\
y_{21}+y_{23}-\beta y_{12}-\beta y_{32} & =0 \\
y_{32}+y_{34}-\beta y_{13}-\beta y_{23}-\beta y_{43} & =0 \\
y_{41}+y_{43}-\beta y_{14}-\beta y_{34} & =0 \\
y_{12}+y_{13}+y_{14} & =\frac{1}{1-\beta^{4}}
\end{aligned}
$$

$y_{12}, y_{13}, y_{14}, y_{21}, y_{23}, y_{32}, y_{34}, y_{41}, y_{43} \geq 0$ 


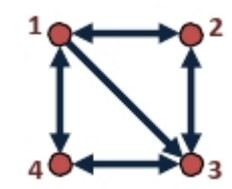

Figure 1: A directed Hamiltonian graph on four nodes

It is easy to check that

$$
\left\{\begin{array}{l}
y_{12}=\frac{1}{1-\beta^{4}}, y_{23}=\frac{\beta}{1-\beta^{4}}, y_{34}=\frac{\beta^{2}}{1-\beta^{4}}, y_{41}=\frac{\beta^{3}}{1-\beta^{4}} \\
y_{13}=y_{14}=y_{21}=y_{32}=y_{43}=0
\end{array}\right.
$$

is a feasible solution of above constraints. Furthermore, this solution is associated with the Hamiltonian policy

$$
\left\{\begin{array}{l}
f(1,2)=1, f(2,3)=1, f(3,4)=1, f(4,1)=1 \\
f(1,3)=f(1,4)=f(2,1)=f(3,2)=f(4,3)=0
\end{array},\right.
$$

that traces out the standard tour " $1 \rightarrow 2 \rightarrow 3 \rightarrow 4 \rightarrow 1$ ".

Now, suppose that the Hamiltonian graph $G$ on $n$ nodes is given and $P$ is a corresponding Hamiltonian matrix. Similarly to Feinberg's approach [4] to derive Constraints (22)-(24), we can define the following set of constraints:

$$
\boldsymbol{x}^{k}(\beta)^{T}(I-\beta P):=\left(1-\beta^{n}\right) \boldsymbol{e}_{k}^{T} \text { for } k=1,2, \ldots, n,
$$

where $\boldsymbol{e}_{k}$ is a unit vector with one 1 at $k^{t h}$ element 0 elsewhere. By exploiting Proposition 4.9 we have:

$$
\boldsymbol{x}^{k}(\beta)^{T}=\left(1-\beta^{n}\right) \boldsymbol{e}_{k}^{T} R^{-1}(\beta)=\boldsymbol{e}_{k} I+\beta \boldsymbol{e}_{k} P+\beta^{2} \boldsymbol{e}_{k} P^{2}+\cdots+\beta^{n-1} \boldsymbol{e}_{k} P^{n-1} .
$$

Now, one may define a new vector $\boldsymbol{x}_{r}^{k}:=\boldsymbol{e}_{k} P^{r}$ for $r=0,1,2, \ldots, n-1$. Clearly, all components of vector $\boldsymbol{x}_{r}^{k}$ are equal to 0 except that one of them that is equal to 1 . This unique element identifies the $r^{\text {th }}$ node visited on a Hamiltonian cycle starting from node $k$. Hence, we can rewrite $\boldsymbol{x}^{k}(\beta)$ in terms of vectors $\boldsymbol{x}_{r}^{k}$ as follows:

$$
\boldsymbol{x}^{k}(\beta)=\boldsymbol{x}_{0}^{k}+\beta \boldsymbol{x}_{1}^{k}+\beta^{2} \boldsymbol{x}_{2}^{k}+\cdots+\beta^{n-1} \boldsymbol{x}_{n-1}^{k} .
$$

It is readily seen that (26) supports the generic structure of solutions corresponding to Hamiltonian policies given in Theorem 6.1 and developed in [3]. Let us move back to Constraints (25) with the right side of (26) in place of $\boldsymbol{x}^{k}(\beta)$ to obtain

$$
\left(\boldsymbol{x}_{0}^{k}+\beta \boldsymbol{x}_{1}^{k}+\beta^{2} \boldsymbol{x}_{2}^{k}+\cdots+\beta^{n-1} \boldsymbol{x}_{n-1}^{k}\right)^{T} R:=\left(1-\beta^{n}\right) \boldsymbol{e}_{k}^{T} \text { for } k=1,2, \ldots, n .
$$


Or, equivalently,

$$
\begin{aligned}
\boldsymbol{x}_{0}^{k}+ & \beta \\
& \left(\boldsymbol{x}_{1}^{k}-P^{T} \boldsymbol{x}_{0}^{k}\right)+\beta^{2}\left(\boldsymbol{x}_{2}^{k}-P^{T} \boldsymbol{x}_{1}^{k}\right)+\cdots \\
& +\beta^{n-1}\left(\boldsymbol{x}_{n-1}^{k}-P^{T} \boldsymbol{x}_{n-2}^{k}\right)-\beta^{n} P^{T} \boldsymbol{x}_{n-1}^{k}=\boldsymbol{e}_{k}-\beta^{n} \boldsymbol{e}_{k} .
\end{aligned}
$$

Now, by equating coefficients of powers of $\beta$ in both sides of the latter equation, we obtain the following set of linear constraints which is free of the parameter $\beta$ :

$$
\begin{aligned}
\boldsymbol{x}_{0}^{k} & =\boldsymbol{e}_{k} \\
\boldsymbol{x}_{r}^{k}-P^{T} \boldsymbol{x}_{r-1}^{k} & =0 \text { for } r=2,3, \ldots, n-1 \\
P^{T} \boldsymbol{x}_{n-1}^{k} & =\boldsymbol{e}_{k} \cdot
\end{aligned}
$$

Analogous to the way that we derived (21), we can expand (28)-(30) as follows:

$$
\begin{aligned}
\sum_{a \in \mathcal{A}(i)} x_{0, i a}^{k} & =\delta_{k i} \text { for } i, k=1,2, \ldots, n \\
\sum_{a \in \mathcal{A}(i)} x_{r, i a}^{k}-\sum_{b \in \mathcal{B}(i)} x_{r-1, b i}^{k} & =0 \text { for } i, k=1,2, \ldots, n, r=1,2, \ldots, n-1 \\
\sum_{b \in \mathcal{B}(i)} x_{n-1, b i}^{k} & =\delta_{k i} \text { for } i, k=1,2, \ldots, n .
\end{aligned}
$$

Based on the way we define vectors $\boldsymbol{x}_{r}^{k}$, we now have an interesting interpretation for components $x_{r, i a}^{k}$. More precisely, if these vectors indeed came from a Hamiltonian transition matrix $P$, then we may demand that $x_{r, i a}^{k}$ be equal to 1 if arc $(i, a)$ is visited at the $r^{t h}$ step of a Hamiltonian cycle starting from node $k$ and otherwise equal to 0 . However, due to complexity associated with the introduction of 0-1 variables, we can relax them to vary between 0 and 1 and try to establish new linear constraints designed to induce them to behave somewhat like $0-1$ variables. New constraints which can be augmented by utilizing this interpretation are listed below:

(i) If a node $i$ is visited at $r^{\text {th }}$ step of a Hamiltonian cycle starting from node $k$, then on the same tour, node $k$ should be visited at $n-r$ steps starting from node $i$ :

$$
\sum_{a \in \mathcal{A}(i)} x_{r, i a}^{k}-\sum_{a \in \mathcal{A}(k)} x_{n-r, k a}^{i}=0 \text { for } i, k=1,2, \ldots, n, i \neq k, r=1,2, \ldots, n-1
$$

(ii) If an arc $(i, a)$ belongs to a particular Hamiltonian cycle, then it should be visited from any starting node on that tour, in particular from starting nodes $k$ and $j$ :

$$
\sum_{r=0}^{n-1} x_{r, i a}^{k}-\sum_{r=0}^{n-1} x_{r, i a}^{j}=0 \text { for } i, k, j=1,2, \ldots, n, k<j, a \in \mathcal{A}(i)
$$


(iii) If an arc $(i, a)$ belongs to a particular Hamiltonian cycle, then based on the starting node, it can be visited at any steps of $0,1, \ldots, n-1$ on that tour:

$$
\sum_{k=1}^{n} x_{r, i a}^{k}-\sum_{k=1}^{n} x_{t, i a}^{k}=0 \text { for } i=1,2, \ldots, n, r, t=0,1, \ldots, n-1, r<t, a \in \mathcal{A}(i)
$$

Of course, if $(i, a)$ does not belong to a Hamiltonian cycle, both terms on the left side of (36) are 0.

(iv) Starting from node $k$, we must visit node $i$ at some step in the next $n-1$ steps:

$$
\sum_{r=0}^{n-1} \sum_{a \in \mathcal{A}(i)} x_{r, i a}^{k}=1 \text { for } i, k=1,2, \ldots, n \text {; }
$$

(v) Starting from node $k$, we must visit exactly one node at $r^{\text {th }}$ step:

$$
\sum_{i=1}^{n} \sum_{a \in \mathcal{A}(i)} x_{r, i a}^{k}=1 \text { for } k=1,2, \ldots, n, r=0,1, \ldots, n-1 ;
$$

(vi) In order to tighten the whole feasible region, we can exploit the notion of a "shortest path". For this purpose, let us assign length 1 to all arcs of graph $G$. If $\theta_{k i}$ is the length of the shortest path between nodes $k$ and $i$ in graph $G$, starting from node $k$, node $i$ cannot be visited earlier than $\theta_{k i}$ steps. Moreover, starting from node $k$, node $i$ cannot be visited later than $n-\theta_{i k}$ steps (on a Hamiltonian cycle), as well:

$$
x_{r, i a}^{k}=0 \text { for } k, i=1,2, \ldots, n, a \in \mathcal{A}(i), r<\theta_{k i}, r \geq n-\theta_{a k} ;
$$

(vii) If $k$ is the starting node, it should not be revisited before the last step:

$$
\begin{gathered}
x_{r, k a}^{k}=0 \text { for } k=1,2, \ldots, n, a \in \mathcal{A}(k), r=1,2, \ldots, n-1 \\
x_{r, b k}^{k}=0 \text { for } k=1,2, \ldots, n, b \in \mathcal{B}(k), r=0,1, \ldots, n-2 ;
\end{gathered}
$$

(viii) Finally, all variables should be non-negative:

$$
x_{r, i a}^{k} \geq 0 \text { for } i, k=1,2, \ldots, n, r=0,1, \ldots, n-1, a \in \mathcal{A}(i) .
$$

Let us denote the polytope defined by the set of linear Constraints $(31)-(42), \mathcal{P} \mathcal{F}$. This model comprises " $n^{2}|\mathcal{A}|$ " variables and " $2 n^{3}+n^{2}+n+n(n-1)|\mathcal{A}|$ " constraints (excluding non-negativity). Therefore, the number of variables and constraints are bounded above by $n^{4}-n^{3}$ and $n^{4}+2 n^{2}+n$, respectively. Moreover, for the special family of cubic graphs ${ }^{3}$, the number of variables and constraints will be $3 n^{3}$ and $5 n^{3}-2 n^{2}+n$, respectively.

Clearly, if we force all $x_{r, i a}^{k}$ variables to be binary variables, then the polytope $\mathcal{P} \mathcal{F}$ will be infeasible for all non-Hamiltonian graphs. However, we hope that by relaxing such $0-1$ condition, this model could still recognize non-Hamiltonian graphs by showing infeasibility.

\footnotetext{
${ }^{3} \mathrm{~A}$ graph is called cubic, if degrees of all its nodes are equal to three.
} 


\begin{tabular}{|c|c|c|c|c|c|}
\hline$n$ & Number of Bridge Graphs & Eliminated & Number of NBNH & Eliminated & Ratio \\
\hline 10 & 1 & 1 & 1 & 0 & 0 \\
\hline 12 & 4 & 4 & 1 & 0 & 0 \\
\hline 14 & 29 & 29 & 6 & 1 & 0.167 \\
\hline 16 & 186 & 186 & 33 & 6 & 0.182 \\
\hline 18 & 1435 & 1435 & 231 & 42 & 0.182 \\
\hline
\end{tabular}

Table 1: Solving HCP for non-Hamiltonian cubic graphs varying from 10-18 nodes

\subsection{Numerical Results}

We have tested several Hamiltonian and non-Hamiltonian cubic graphs through polytope $\mathcal{P F}$. So far, it has been successful in recognizing all bridge cubic graphs ${ }^{4}$ on $10,12,14$, 16 and 18 nodes by showing infeasibility. Surprisingly, perhaps, it could also eliminate some of the non-bridge non-Hamiltonian cubic graphs (NBNH for short) by showing infeasibility. Table 1 displays the result of these experiments.

In Table 1, from left, the first column shows the number of nodes in the family of cubic graphs, the second column indicates the total number of bridge graphs in that particular family, the third columns displays the number of such bridge graphs which made the corresponding polytope $\mathcal{P} \mathcal{F}$ infeasible, the fourth column indicates the total number of NBNH graphs in that particular family, the fifth columns displays the number of such NHNB graphs which made the corresponding polytope $\mathcal{P} \mathcal{F}$ infeasible and the last column gives the ratio of the fifth column over the fourth one.

Acknowledgment This research was partially supported by ARC Grant DP0666632. The second author was visiting INRIA in Sophia Antipolis, France, while working on this paper. We are indebted to Vladimir Ejov and Giang Nguyen for many useful discussions and for supplying the original (but different) proof of the identity in Proposition 5.3.

\section{References}

[1] K.E. Avrachenkov, J.A. Filar and P.G. Howlett, Analytic Perturbation Theory and Its Applications, SIAM Publisher, 2013.

[2] V.S. Borkar, V. Ejov and J. Filar, "On the Hamiltonicity Gap and Doubly Stochastic Matrices", Random Structures \& Algorithms, 34(4):502-519, 2009.

\footnotetext{
${ }^{4} \mathrm{~A}$ graph is called a bridge graph, if the set $\mathcal{V}$ can be partitioned into two non-empty sets such that there is only one arc from one partition to the other one.
} 
[3] A. Eshragh, J.A. Filar and M. Haythorpe, "A Hybrid Simulation-Optimization Algorithm for the Hamiltonian Cycle Problem", Annals of Operations Research, 189(1):103-125, 2011.

[4] E.A. Feinberg, "Constrained Discounted Markov Decision Processes and Hamiltonian Cycles", Mathematics of Operations Research, 25(1):130-140, 2000.

[5] J.A. Filar and D. Krass, "Hamiltonian Cycles and Markov Chains", Mathematics of Operations Research, 19(1):223-237, 1994.

[6] J.A. Filar, "Controlled Markov Chains, Graphs \& Hamiltonicity", Foundation and Trends ${ }^{\circledR}$ in Stochastic Systems, 1(2):77-162, 2006.

[7] M.R. Garey and D.S. Johnson, Computers and Intractability: A Guide to the Theory of NP-Completeness, W.H. Freeman \& Co., New York, 1979.

[8] J.J. Hunter, "Stationary Distributions and Mean First Passage Times of Perturbed Markov Chains", Linear Algebra and its Applications, 401:217-247, 2005.

[9] J.G. Kemeny and J.L. Snell, Finite Markov Chains, van Nostrand, New York, 1960.

[10] M.L. Puterman, Markov Decision Processes: Discrete Stochastic Dynamic Programming, Wiley-Interscience, $1^{\text {st }}$ Edition, New Jersey, 2005. 